\title{
Domain structures during magnetization reversal in exchange-biased layers
}

\author{
A. Kirilyuk and Th. Rasing \\ Research Institute for Materials, University of Nijmegen, Toernooiveld 1, 6525 ED Nijmegen, \\ The Netherlands \\ H. Jaffrès, D. Lacour, and F. Nguyen Van Dau \\ Unité Mixte de Physique CNRS/Thales, Domaine de Corebeville, Orsay, France
}

The magnetization reversal of an exchange-biased $\mathrm{Co} / \mathrm{NiO}$ layer is studied with the help of magneto-optical microscopy, as a function of the angle between the applied magnetic field and the biasing direction. Based on domain patterns, a model of the magnetization reversal in these layers is presented. The drastic changes in the domain patterns indicate different domain nucleation conditions for different directions of the effective field. (C) 2002 American Institute of Physics.

[DOI: $10.1063 / 1.1454978$ ]

The exchange-biased antiferromagnet/ferromagnet bilayers are very interesting from both technological as well as fundamental point of view. When cooled down in an applied magnetic field through the Neél temperature of the antiferromagnet $T_{N}\left(T_{N}<T_{C}\right.$, where $T_{C}$ is the Curie temperature of the ferromagnetic layer), the hysteresis of the ferromagnet becomes asymmetrically shifted from zero field. In other words, the anisotropy of the ferromagnetic layer becomes unidirectional, ${ }^{1,2}$ its coercivity being increased as well. Several possible mechanisms have been invoked to explain the increased coercivity in exchange-bias systems, such as irreversible switching of the antiferromagnet of the antiferromagnet or inhomogeneous reversal of the ferromagnet. ${ }^{3}$

Ideally speaking, the unidirectional anisotropy should lead to the absence of magnetic domains in such a structure. Indeed, with only one equilibrium azimuthal angle of $\mathbf{M}$, the whole sample would rotate coherently into the effective field direction. This is in most cases contrary to the experimental observation of magnetic domains in such layers, ${ }^{4-6}$ although for some cases, a coherent magnetization rotation has been also reported. ${ }^{7}$ Measurements of the exchange bias and coercive field as a function of the applied field angle ${ }^{8,9}$ are very informative and provide a good test for the validity of existing theoretical models. ${ }^{10}$

This article presents a study of the domain patterns during the magnetization reversal in exchange-biased $\mathrm{Co} / \mathrm{NiO}$ bilayers, for different angles between the applied magnetic field and the exchange biasing axis. In some way, our measurements are similar to what has been done for the free ferromagnetic layer in spin-valve structures, ${ }^{4}$ with a corresponding theoretical model. ${ }^{11}$ The influence of the interface between the ferromagnet and antiferromagnet, however, can only be studied via the reversal of the pinned layer. Domain patterns have been shown to exist in all investigated configurations. In some cases, the whole sample area is reversed by a single high-mobility domain wall producing a very sharp edge of the hysteresis loop. We present the complete picture of domain formation in the magnetization reversal process and further discuss the influence of the balance between unidirectional and uniaxial anisotropy and coercivity.

Our samples were sputtered Co layers on top of $\mathrm{NiO}$, with a full structure of $\mathrm{Si}(100) / / \mathrm{NiO}(300 \AA) / \mathrm{Co}(150 \AA) /$ $\mathrm{Au}(50 \AA)$. To create the bias, they were post-annealed to $300{ }^{\circ} \mathrm{C}$ in an applied magnetic field.

The magnetization reversal was studied with the help of spatially resolved magneto-optical Kerr effect (MOKE) as well as with magnetization-induced second harmonic generation (MSHG). The setup was built in such a way that both effects could be measured in the same configuration and at the same spot on the sample. For convenience, the transverse magneto-optical geometry was used. The MOKE hysteresis loops were measured from the same spot (by size and position) where the images were taken, approximately $300 \mu \mathrm{m}$ in diameter.

For the light wavelength of $800 \mathrm{~nm}$ the amplitude of the Kerr rotation in this configuration was $2 \Theta_{\mathrm{Kerr}}=70 \mathrm{mdeg}$. To obtain a visible magnetic contrast with such small rotation, subtraction of the nonmagnetic background is required. Therefore, the imaging was done in the following way: (a) saturating the sample in one direction; (b) taking the reference image; (c) applying the field in the opposite direction, with an amplitude roughly equal to that of the coercive field; (d) after a given time (5-500 s) the field was switched to a value roughly in the middle of the hysteresis loop, to achieve a metastable state; (e) taking the image of the domain pattern in this state; and (f) making subtraction of the reference image. In this way the images corresponding to various parts of the hysteresis loop could be obtained.

It was also possible to record the images directly on the hysteresis loop. Though showing domain patterns, such images were of very low quality because of magnetic aftereffect in these samples (slow domain motion in applied field) 


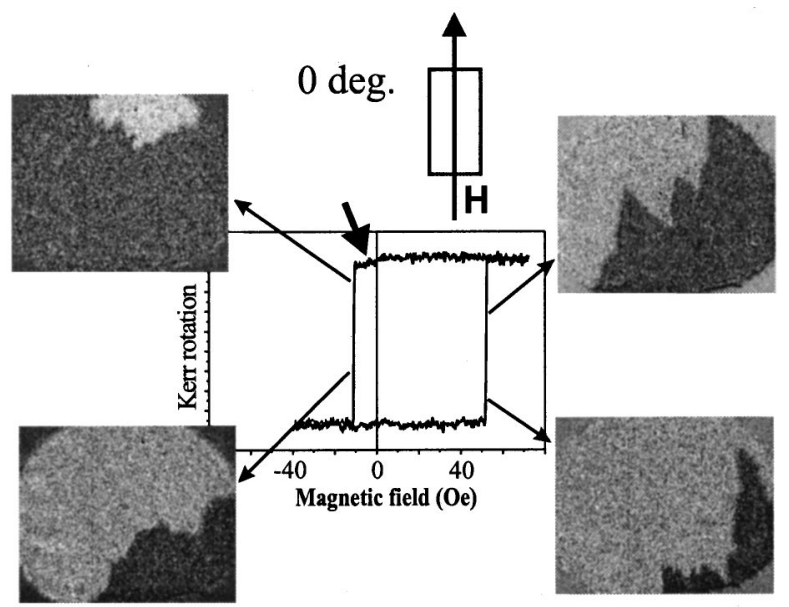

FIG. 1. Magnetization hysteresis and domain pattern for the case when the magnetic field is applied along the exchange-biasing axis.

that restricted the image acquisition time. It was evident, however, that the subsequent field reduction to the center of the hysteresis loop did not change the patterns but made them (meta)stable.

Figure 1 shows the magnetization hysteresis loop measured with MOKE in the applied field parallel to the exchange-biasing axis. The shape of the loop is extremely square which can be a consequence of either a coherent magnetization flip (such as in monodomain particles) or of the presence of high-mobility domain walls. Our measurements (see images in Fig. 1) clearly demonstrate the latter case. On these images, white (black) domain corresponds to the up (down) magnetization direction. Small deviations from this vertical axis are not distinguished.

Except for the difference in the domain wall shape the pattern is quite similar for both magnetization reversal directions: domains are very large so at the scale of the image $(300 \mu \mathrm{m})$ we observe a single domain wall running through the sample (compare the opposite case in Ref. 6). We have also an indication that the whole sample are (approximately $4 \times 8 \mathrm{~mm}^{2}$ ) is reversed by a single domain wall nucleated somewhere at the edge. Such a behavior is in perfect agreement with the square hysteresis loop. The difference in the domain wall shape that is seen at the images (randomly curved in an easy direction and a sort of "zig-zag" structure in the hard one), although reproducible, is not clear at that point.

There is one little feature, however, that is visible in the hysteresis loop but not appearing in the images: when the magnetization is saturated opposite to the bias direction and then the field is sweeped back to the "easy" side, there is a little dip in the magnetization before the actual reversal takes place (shown by an arrow in Fig. 1). As far as the imaging is concerned, no change is observed at that point.

When the applied magnetic field has an angle $\theta$ with the biasing axis, the magnetization pattern changes (see Figs. 2 and 3). While the magnetization reversal into the easy direction still takes place via the motion of extended domain walls (at least for smaller values of $\theta, \theta \leqslant 20^{\circ}$, see Figs. 2 and 3), the domains become smaller in the opposite direction. With
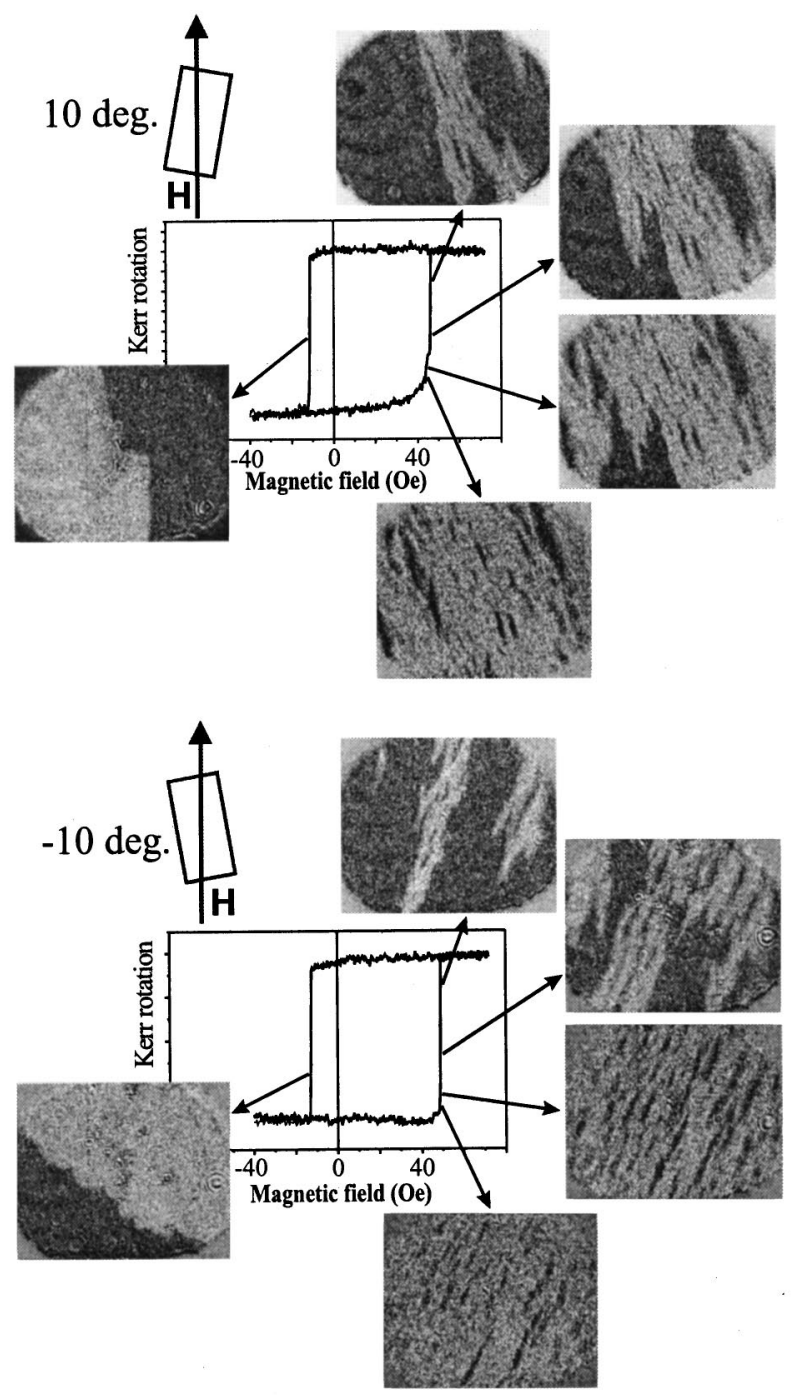

FIG. 2. Magnetization hysteresis and domain pattern for the case when the magnetic field is applied at an angle of $\pm 10^{\circ}$ with respect to the exchangebiasing axis.

that, a characteristic stripe pattern appears on the images. An interesting point is that the stripe pattern does not align with the sample exchange-biasing axis. Indeed, Fig. 2 clearly shows that while the sample is rotated clockwise, the pattern seems to be rotated counterclockwise, and vice versa.

The hysteresis loop shape changes as well (Figs. 2 and 3 ). For the positive angle values, the right-bottom corner becomes rounded while for the negative ones, a kind of a kink appears. Such a change can be explained by an appearance of a magnetization component perpendicular to the applied field, i.e., in the plane of light incidence. The direction of this component depends on the sign of the angle and therefore in one case, it adds to the total magneto-optical signal and in the other, it subtracts from it. MSHG measurements were used to confirm this "longitudinal" component. Indeed, the MSHG intensity in the $P_{\text {in }} S_{\text {out }}$ polarization combination is zero unless there is a component of $\mathbf{M}$ in the plane of incidence. ${ }^{12}$ The hysteresis loop measured in that configuration (see Fig. 3) clearly shows an increase of MSHG intensity corresponding to the magnetization tilt 


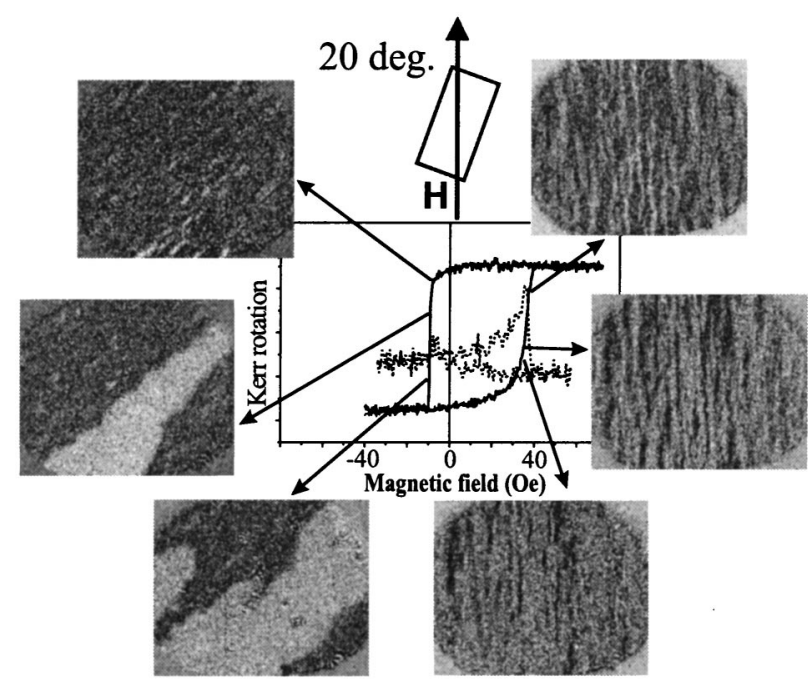

FIG. 3. Magnetization hysteresis and domain pattern for the case when the magnetic field is applied at an angle of $20^{\circ}$ with respect to the exchangebiasing axis. Dotted line shows the MSHG intensity hysteresis in $P_{\text {in }} P_{\text {out }}$ polarization combination.

away from the field axis. A complete analysis of MSHG data will be published elsewhere. ${ }^{13}$

At larger angles $\theta$ the domains become smaller and smaller. In addition, the domain pattern is not stable anymore so at some point (at angles of more than $40^{\circ}$ ) the imaging becomes impossible, from both resolution (of the order of $2-3 \mu \mathrm{m})$ and contrast point of view. For the field direction orthogonal to the exchange-biasing axis we were not able to visualize any pattern whatsoever.

We can summarize the magnetization reversal in exchange-biased layer in the following way. After the sample has been saturated into the easy direction, the application of the opposite magnetic field along the biasing axis leads to a creation of a single domain wall that quickly sweeps across the sample. As a result, the sample becomes saturated into the "hard" direction. Afterwards the field is swept across the hysteresis loop again; a slight kink in the hysteresis loop indicates a partial relaxation of the magnetization. No domains can be observed at this point, however. After the field reaches the "positive" coercive field value, the magnetization is finally reversed again via a formation of a large-scale domain wall. As we have already mentioned, the shape of the domain wall is different for the positive and "negative" reversals. This difference can probably be explained by the difference in effective magnetic field during the reversal.

The kink remains unclear; the formation of tiny oppositely magnetized magnetic domains (not visible on the images) can be excluded because otherwise such domains would serve as the nucleation centers during reversal; the presence of a single domain wall is in clear contradiction with this assumption. The MSHG data (see Fig. 3 and Ref. 13) clearly show that magnetization may deviate from the field axis also at this point. In this case, however, it happens also with the applied field parallel to the exchange-biasing axis.

When the applied field constitutes an angle with the biasing axis, the magnetization reversal changes. First, when field is swept from the negative saturation value to the positive side, there is an obvious relaxation of the magnetization into the easy direction, i.e., the coherent rotation of the sample magnetization. Then, at a positive coercive field, the reversal takes off by formation of stripe domains directed at some angle with both the applied field and the sample axis. It may be supposed that the domains are formed along the effective field in the sample (i.e., the effective field when the applied field is given by the positive coercive field value). This is a tricky point, however: if the magnetization was already tilted to that side, why should the system bother to create domains at all?

Afterwards the magnetization is finally saturated to the positive direction.

Reversing the field again allows us to observe the kink on the hysteresis loop without revealing anything on the domain pattern. Also as in the previous case, we observe a tilt of the magnetization from the field axis (see small bump of the MSHG intensity in Fig. 3). After this tilt, the formation of domains occurs that can be large for small azimuthal angles (up to $20^{\circ}$ ) and stripelike with preferable orientation roughly along the biasing axis for large ones. Finally the magnetization is saturated back into the positive direction.

To conclude, the observation of the domain patterns during the magnetization reversal in exchange-biased magnetic layers confirmed the domain formation mechanism as the dominating one. The coherent magnetization reversal was found to be responsible for a small part of the total magnetization change.

Part of this work was supported by the Brite Euram program TunnelSense and the European TMR network NOMOKE.

${ }^{1}$ W. H. Meiklejohn and C. P. Bean, Phys. Rev. 102, 1413 (1956).

${ }^{2}$ For recent review see J. Nogués and I. K. Schuller, J. Magn. Magn. Mater. 192, 203 (1999); A. E. Berkowitz and K. Takano, ibid. 200, 552 (1999).

${ }^{3}$ M. D. Stiles and R. D. McMichael, Phys. Rev. B 63, 064405 (2001).

${ }^{4}$ J. P. King, J. N. Chapman, J. C. S. Kools, and M. F. Gilles, J. Phys. D 32, 1087 (1999).

${ }^{5}$ V. I. Nikitenko, V. S. Gornakov, A. J. Shapiro, R. D. Shull, K. Liu, S. M. Zhou, and C. L. Chien, Phys. Rev. Lett. 84, 765 (2000).

${ }^{6}$ D. X. Yang, H. D. Chopra, P. J. Chen, H. J. Brown, L. J. Swartzendruber, and W. F. Egelhoff, Jr., J. Phys. Soc. Jpn. 87, 4942 (2000).

${ }^{7}$ C. Leighton, M. R. Fitzsimmons, P. Yashar, A. Hoffmann, J. Nogués, J. Dura, C. F. Majkrzak, and I. K. Schuller, Phys. Rev. Lett. 86, 4394 (2001).

${ }^{8}$ T. Ambrose, R. L. Sommer, and C. L. Chien, Phys. Rev. B 56, 83 (1997).

${ }^{9}$ H. Xi, M. H. Kryder, and R. M. White, Appl. Phys. Lett. 74, 2687 (1999); H. Xi and R. M. White, J. Appl. Phys. 86, 5169 (1999).

${ }^{10}$ J. Geshev, L. G. Pereira, and J. E. Schmidt, Phys. Rev. B 64, 184411 (2001).

${ }^{11}$ M. Labrune, J. C. S. Kools, and A. Thiaville, J. Magn. Magn. Mater. 171, 1 (1997)

${ }^{12}$ B. Koopmans, M. Groot Koerkamp, Th. Rasing, and H. van den Berg, Phys. Rev. Lett. 74, 3692 (1995).

${ }^{13}$ A. Kirilyuk, Th. Rasing, H. Jaffrès, D. Lacour, and F. Nguyen Van Dau (to be published). 